\title{
The Apollo Archive Explorer
}

\author{
Douglas W. Oard \\ College of Information Studies/UMIACS \\ University of Maryland, College Park, MD USA \\ oard@umd.edu
}

\author{
Joseph Malionek \\ Eleanor Roosevelt High School \\ Greenbelt, MD USA \\ jmalionek@gmail.com
}

\begin{abstract}
A system for exploring the rich recorded legacy of the Apollo missions to the Moon, using the event structure of each mission as an organizing principle, will be demonstrated. A scalable implementation is achieved by automating temporal, spatial, and topical content alignment across diverse media. Multiple access points are supported, including event-based access through the flight plan, time-based access using event timelines, and contentbased access using information retrieval techniques.
\end{abstract}

\section{Categories and Subject Descriptors}

H.3.7 [Information Systems]: Information Storage and Retrieval digital libraries.

\section{General Terms}

Design, Human Factors.

\section{Keywords}

Information integration, streaming media, archival sources.

\section{INTRODUCTION}

In a four-year period between December 1968 and December 1972, Twenty-four Americans traveled to Earth's Moon; twelve of them landed. It is one of the most extensively documented events in human history. Paradoxically, no one knows what happened. Many people know a little and some people know a lot, but nobody knows the whole story. Of course, that's the case for any complex undertaking, and it is why making sense of the historical record requires consultation of multiple sources. As more primary source materials come online, new opportunities emerge to support analysis and synthesis of the historical record. The Apollo missions offer an outstanding testbed for applying time-synchronized event reconstruction to archival materials because several large collections of primary source materials from the Apollo program are online, a substantial subset of that record includes sufficiently precise timing information for automatic alignment, and individual records (documents, audio recordings, and still and moving images) tell only a part of the story. This paper describes our work to date with some of those materials.

\section{THE LEGACY OF APOLLO}

At the dawn of the Web in the early 1990's, Eric Jones created the Apollo Lunar Surface Journals (ALSJ), a set of annotated radio

\footnotetext{
Permission to make digital or hard copies of all or part of this work for personal or classroom use is granted without fee provided that copies are not made or distributed for profit or commercial advantage and that copies bear this notice and the full citation on the first page. Copyrights for third-party components of this work must be honored. For all other uses, contact the owner/author(s).

JCDL'13, July 22-26, 2013, Indianapolis, Indiana, USA.

ACM 978-1-4503-2077-1/13/07.
}

transcripts for the lunar surface period of each mission. He (and many others) scanned the typewritten originals, performed OCR, manually corrected the resulting errors, formatted the result using HTML, and then interviewed ten of the twelve moonwalkers to obtain commentary and clarification [1]. The idea caught on, and Apollo Flight Journals (AFJ) are now being created to provide a similar presentation for the other parts of the Apollo missions.

In addition to the transcribed radio communications in the Apollo Lunar Surface Journals, transcripts of onboard recordings are also available. The Apollo program used two crewed spacecraft, one of which (the Lunar Module or LM) was designed to land on the moon; the second spacecraft (the Command Module or CM) remained in lunar orbit awaiting the LM's return from the surface. Each contained a combined voice and data recorder which were later transcribed. The idea for the Apollo Archive Explorer started with the release of a complete set of Apollo mission transcripts in 2001 [4].

Video from the Apollo missions is available from three sources: Earth-based cameras (for launch and splashdown), television signals transmitted to earth during the mission, and video filmed during the mission and developed upon return to Earth. Along with recording video, the Apollo astronauts also took about 6,000 still photographs using Hasselblad cameras which were usually mounted on the astronaut's chest, yielding an unmatched sequence of first person views of lunar surface operations.

Oral history interviews are a useful source that can help fill gaps in the documentary record of the Apollo program. The Johnson Space Center oral history project has interviewed a multitude of people from the Apollo missions, and transcripts from almost all of those interviews are available online in PDF format. Audio from those interviews can also be ordered (for a modest fee, in CD format) from the University of Houston, Clear Lake.

\section{THE APOLLO ARCHIVE EXPLORER}

The design goal of the Apollo Archive Explorer is to provide rich experiential access to the records of what actually happened during the Apollo missions to the Moon. By "experiential" access, we mean that we seek to provide a richer experience than could be achieved with individual sources. Our focus on "what actually happened" places a primacy on the events during a mission, rather than on design issues, mission planning, or training. "Rich access" has a second meaning here as well-users of the system should be able to get to the reconstruction of an event in ways that best suit their thought process.

Figure 1 depicts the user interface of the Apollo Archive Explorer, which is implemented as a Java application. The screen is divided into four regions: control (upper left), images (upper right), and two tab-selectable document display zones (lower left and lower right). The top row of the control region displays the Ground Elapsed Time (GET) since launch from Earth for the present point in the replay along with a "Pause" button, selection of the mission to be displayed (presently Apollo 11, 14, 15, 16, or 


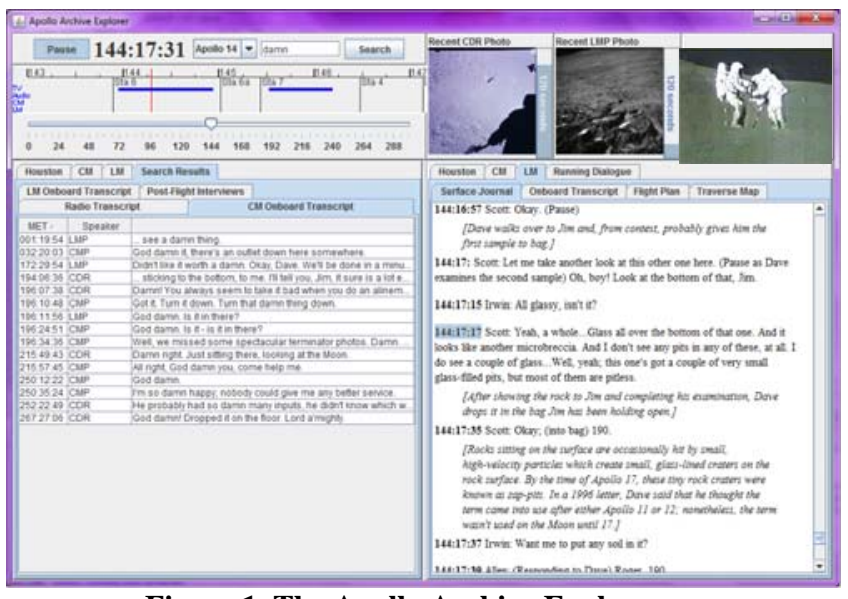

Figure 1. The Apollo Archive Explorer. region of the display and then clicked on the "Search" button. That action automatically replaces the lower left document display area with the search results. Results from any one of the scanned transcripts (CM recorder, LM recorder, radio) or the oral history interviews can then be examined by selecting the appropriate tab. Selecting any line in a search result from a scanned transcript will reset the ground elapsed time to the time associated with that result. The search function is implemented using the Lucene text retrieval system. The text of each utterance in each scanned transcript is indexed in advance, thus yielding rapid response to queries at run time. The most significant innovation required to build the Apollo Archive Explorer was the ability to represent structural elements of scanned transcripts in ways that allowed effective time synchronization and contentbased search. We modified the Bilingual Resource Inference and Dictionary Generation Environment (BRIDGE) system, which had originally been developed to extract translation lexicons from bilingual dictionaries, to perform this structural analysis [4].

\section{CONCLUSION}

The policy of the U.S. National Archives for appraisal of records for retention states, in part: "The future research potential of records is the most difficult variable to determine. What is of relatively low research use today may become of great research use in the future. Perhaps even more important and difficult to predict are the issues and topics that will be considered of significance in the future. Nevertheless, it is important to consider this question in making appraisal decisions. It is necessary to consider the kinds and extent of current research use and to try to make inferences about anticipated use both by the public and by the Government.” [3]

Envisioning the kinds of richly textured event alignment that the Apollo Archive Explorer demonstrates would have been challenging forty years ago in an era when even the simple computers that flew to the Moon cost \$46 million to design and build. As a result, some sources that we could now productively integrate simply are not available today in any known location. By building systems such as the Apollo Archive Explorer, we can help to inform tomorrow's records retention decisions (e.g., from the extensive records generated by the 135 Space Shuttle missions).

\section{ACKNOWLEDGMENTS}

This material is based upon work supported by NSF under Grant 1218159. Opinions, findings and conclusions or recommendations expressed in this material are those of the authors and do not necessarily reflect the views of the National Science Foundation.

\section{REFERENCES}

[1] Heiken, G. \& Jones, E. (2007). On the Moon: The Apollo Journals, Springer Praxis.

[2] Ma, H., Doermann, D., Karagol-Ayan, B., Oard, D. \& Wang, J. (2004). "Parsing and Tagging Bilingual Dictionaries," Traitement Automatique des Languages, 44(2)125-150.

[3] National Archives and Records Administration (2007). Appraisal Policy of the National Archives and Records Administration, NARA Directive 1441, September.

[4] Swanson, G. (2001). "We Have Liftoff!: The Story behind the Mercury, Gemini and Apollo Air to Ground Transmissions," Spaceflight, vol. 43, Feb.
The results of conducting a text-based search of the scanned has entered a term in the query box at the top right of the control 